\title{
recildunds
}

Revista Cientifica Mundo de la Investigación y el Conocimiento

\author{
Marola Narcisa Beltrán Mora a; Víctor Martín Parrales Carvajal ${ }^{\text {b}}$; Gerson Damacio \\ Ledesma Álvarez ${ }^{\mathrm{c}}$
}

El Buyer Persona como factor clave entre las tendencias en Gestión Empresarial

The Buyer Person as a key factor among the trends in Business Management

Revista Científica Mundo de la Investigación y el Conocimiento. Vol. 3 núm.3. Esp., noviembre, ISSN: 2588-073X, 2019, pp. 659-681

DOI: 10.26820/recimundo/3.(3.Esp).noviembre.2019.659-681

URL: http://recimundo.com/index.php/es/article/view/615

Código UNESCO: 5311 Organización y Dirección de Empresas

Tipo de Investigación: Artículo de Revisión

(C) RECIMUNDO; Editorial Saberes del Conocimiento, 2019

Recibido: 15/09/2019 Aceptado: 23/10/2019 Publicado: 30/11/2019

Correspondencia: mbeltranm@utb.edu.ec

a. Magister en Economía y Dirección de Empresas; Ingeniera Comercial y Empresarial Especialización en Finanzas; Docente Investigador de la Facultad de Administración y Finanzas; Universidad Técnica de Babahoyo; Babahoyo, Ecuador; mbeltranm@utb.edu.ec

b. Magister en Administración de Empresas; Licenciado en Ciencias de la Educación en la Especialización de Comercio y Administración; Profesor de Segunda Enseñanza en la Especialización de Comercio y Administración; Docente Investigador de la Facultad de Administración y Finanzas; Universidad Técnica de Babahoyo; Babahoyo, Ecuador; vparralesc@utb.edu.ec

c. Master Universitario en Formación Internacional Especializada del Profesorado Especialidad en Orientación Educativa; Licenciado en Ciencias de la Educación en la Especialización de Educación Primaria; Licenciado en Ciencias Económicas; Universidad Técnica de Babahoyo; Docente Investigador de la Facultad de Administración y Finanzas; Babahoyo, Ecuador. 


\section{El Buyer Persona como factor clave entre las tendencias en Gestión Empresarial}

Vol. 3, núm. 3 Esp., (2019)

Marola Narcisa Beltrán Mora; Víctor Martín Parrales Carvajal; Gerson Damacio Ledesma Álvarez

\section{RESUMEN}

Desde un principio, la gestión empresarial ha sido el escenario que se encarga de identificar y determinar en las empresas las diferentes estrategias que persiguen su crecimiento, haciéndolas capaces de asumir los retos de la competitividad actual que les permitirá la supervivencia ante la globalización. A nivel de mercadeo, la perspectiva en la experiencia del cliente es considerada una de las mayores tendencias de gestión empresarial que cada día adquiere mayor relevancia. En la actualidad, las estrategias de marketing digital optan por crear perfiles de buyer personas. En el marco de esta contextualización es que ha surgido el interés por desarrollar la presente investigación, que por propósito tiene la revisión y recopilación de información reciente contenida y disponible de manera libre en bases de datos previamente establecidas, relacionada fundamentalmente con el buyer persona como parte de las estrategias que han tenido relevancia en el contexto de la gestión empresarial. Dentro de los resultados obtenidos se pudo constatar que, un Buyer Persona resulta ser un elemento clave previo al establecimiento de estrategias de Inbound Marketing, ya que es indiscutiblemente es parte elemental en la obtención de mejores resultados. Se concluye acompañando la creencia de que la ideología del inbound representará una de las maniobras más sostenibles a largo plazo en un futuro próximo, ya que, independientemente de la que acelerada evolución del consumidor, esa valiosa información significará posiblemente en una de las muy pocas formas de construir relaciones comerciales respetables y de estar al tanto de las empresas.

Palabras Claves: Competitividad; Experiencia del Cliente; Marketing Digital; Perfiles; Inbound Marketing. 


\title{
El Buyer Persona como factor clave entre las tendencias en Gestión \\ Empresarial
}

Vol. 3, núm. 3 Esp., (2019)

Marola Narcisa Beltrán Mora; Víctor Martín Parrales Carvajal; Gerson Damacio Ledesma

Álvarez

\begin{abstract}
From the beginning, business management has been the scenario that identifies and determines in companies the different strategies that pursue their growth, making them capable of taking on the challenges of current competitiveness that will allow them to survive in the face of globalization. At the marketing level, the customer experience perspective is considered one of the biggest business management trends that every day acquires greater relevance. Currently, digital marketing strategies choose to create profiles of buyer people. Within the context of this contextualization, interest in developing this research has arisen, which by the way has the review and collection of recent information freely contained and available in previously established databases, primarily related to the buyer person as part of the strategies that have been relevant in the context of business management. Among the results obtained, it was found that a Buyer Persona turns out to be a key element prior to the establishment of Inbound Marketing strategies, since it is indisputably an elementary part in obtaining better results. It is concluded by accompanying the belief that the inbound ideology will represent one of the most sustainable long-term maneuvers in the near future, since, regardless of the accelerated evolution of the consumer, that valuable information will possibly mean in one of the very few ways to build respectable business relationships and to be aware of companies.
\end{abstract}

Keywords: Competitiveness; Customer Experience; Digital Marketing; Profiles; Inbound Marketing. 


\section{El Buyer Persona como factor clave entre las tendencias en Gestión Empresarial}

Vol. 3, núm. 3 Esp., (2019)

Marola Narcisa Beltrán Mora; Víctor Martín Parrales Carvajal; Gerson Damacio Ledesma Álvarez

\section{Introducción.}

La gestión empresarial desde el principio de los tiempos ha sido escenario que se encarga de identificar y determinar en las empresas, las diferentes estrategias que persiguen su crecimiento, haciéndolas capaces de asumir los retos de la competitividad actual que les permitirá la supervivencia ante la globalización. (Vargas, Solarte, \& Moreno, 2012).

En el área del Marketing una de las mayores tendencias de gestión empresarial y que cada día adquiere mayor relevancia es el enfoque en la experiencia del cliente. En tal sentido, las empresas deben centrarse en la investigación de la buyer persona como base del proceso de desarrollo de productos.

En el marketing tradicional se define como target al público objetivo a quien se dirigirá la comunicación y que pueden ser nuestros clientes potenciales; sin embargo, esta definición hoy en día se queda muy corta para poder categorizar a los posibles compradores. Por ello, actualmente las estrategias de marketing digital optan por crear perfiles de buyer personas.

Nuviala et al. citados en Alguacil, Crespo, \& Pérez (2019) refieren que, en los últimos tiempos, "las estrategias de marketing se han dirigido a ciertos grupos de compradores de interés para la marca conocidos como targets, alejándose del marketing para todos y segmentando por grupos de usuarios" (p. 139), sin embargo, estos últimos afirman que ese paradigma ha ido cambiando recientemente.

Igualmente, Cafaro (2019) refiere a Navarrete quien en el mismo orden de ideas afirma que "la gran cantidad de información en la red ha generado un cambio en el paradigma de 


\section{El Buyer Persona como factor clave entre las tendencias en Gestión \\ Empresarial}

Vol. 3, núm. 3 Esp., (2019)

Marola Narcisa Beltrán Mora; Víctor Martín Parrales Carvajal; Gerson Damacio Ledesma

Álvarez

ventas: ahora lo importante son las personas y no el producto o servicio". La tratadista explica que eso hace representa a que, en la actualidad, las personas que investigan por internet acerca de sus necesidades, una vez que están listos para realizar la compra optan por recibir una atención personalizada, ya que en ese momento manejan información importante y amplia acerca de la empresa y el producto/servicio que requieren y desean. (p. 74).

El propósito fundamental de esta investigación es revisar y recopilar información reciente contenida en la literatura académico-científica disponible de manera libre en las bases de datos consultadas, relacionada con el buyer persona como una tendencia que forma parte de las estrategias que han tenido relevancia en el contexto de la gestión empresarial. De igual forma, se precisa exponer conceptos asociados tales como gestión empresarial, gestión tecnológica, marketing estratégico, entre otros, que en definitiva faciliten la comprensión de esta temática.

\section{Materiales y Métodos.}

La presente investigación, desarrollada a principios de noviembre de 2019, principalmente se basó en una revisión de la bibliografía científico-académica disponible de manera completa y gratuita mediante el uso de diferentes bases de datos, entre estas: ScienceDirect, Redib, SciELO, Dialnet, Refseek, DOAJ y otras. En estas se determinó como términos y/o expresiones de indagación, lo siguiente: "gestión empresarial", "buyer persona" y "buyer persona como estrategia de gestión empresarial" a fines de explicar los aspectos fundamentales de Buyer Persona y por qué se le considera como una tendencia en el ámbito de gestión empresarial. 


\section{El Buyer Persona como factor clave entre las tendencias en Gestión Empresarial}

Vol. 3, núm. 3 Esp., (2019)

Marola Narcisa Beltrán Mora; Víctor Martín Parrales Carvajal; Gerson Damacio Ledesma Álvarez

Con los resultados obtenidos, debido a que se evidenció la falta de contenido específico que en base a sus títulos identificativos se relacionaran de manera más directa con la temática planteada.

En base a lo antes dicho y al objetivo mencionado, fue igualmente necesario ir filtrando esos resultados, aplicando como criterios de selección: la mayor correlación y/o relevancia temática respecto al objetivo particular; accesibilidad al material de manera completa y gratuita; preferiblemente escritos en español o inglés; con fecha de publicación dentro de los últimos diez años, inclusive el corriente, es decir, de 2009 a 2019; literatura concebida como de tipo artículos científicos en general, tesis de grado, postgrado y doctorado, libros electrónicos, libros físicos digitalizados, presentaciones de conferencias, publicaciones formales u oficiales de instituciones públicas o privadas de reconocida trayectoria en el área administrativa, contable o económica; protocolos, boletines o folletos informativos, notas académicas, ensayos, manuales o instrumentos de procedimiento, y demás trabajos monográficos estructurados con características académicas desarrollados en el marco de la administración de empresas, sin embargo, es posible que algunas fuentes citadas no se correspondan con los criterios antes expuesto, y ello ocurre es porque el equipo consideró hacer ciertas excepciones en base a que tales registros bien podrían asumirse como aún vigentes o con evidencia de alto valor científico.

Se desestimaron editoriales, cartas al editor y otro tipo de materiales bibliográficos que, básicamente, no cumplían con la categoría científico-académico procurada.

De forma secundaria, se realizó una búsqueda sin limitación cronológica ni del nivel de evidencia para identificar información relevante y necesaria para el desarrollo de la presente 


\section{El Buyer Persona como factor clave entre las tendencias en Gestión \\ Empresarial}

Vol. 3, núm. 3 Esp., (2019)

Marola Narcisa Beltrán Mora; Víctor Martín Parrales Carvajal; Gerson Damacio Ledesma

Álvarez

revisión. La inclusión o exclusión de cada una de las referencias en la presente revisión se definió por el consenso de los autores de esta obra, al igual que las decisiones discordantes.

\section{Resultados.}

Gestión empresarial

Este concepto, muy bien podría iniciarse haciendo alusión a fundamentales conceptos que van de la mano, y causalmente estos pueden ser extraídos de la obra de Espejel, Leyva, \& Cavazos (2017).

A manera de preámbulo, estos especialistas se refieren al estudio de Haladay para exponer la globalización es "una realidad del ambiente de negocios del siglo XXI", y complementan alegando que:

(...) los gerentes suelen estar conscientes de las actuales tendencias económicas y de mercado, así como de las obligaciones que tienen de llevar a sus negocios a desarrollar una visión estratégica global, que les permita enfrentar los escenarios probables y tomar las decisiones más idóneas, de tal forma que se solventen los obstáculos que limitan el desempeño eficiente de sus empresas. (p. 2).

Luego, se apoyan en el aporte de Lall, Albadalejo y Mesquita

...el concepto de competitividad proviene de la bibliografía sobre administración de empresas, y viene a ser la base para el análisis estratégico empresarial por lo que ellos mencionan que las compañías compiten para captar mercados y recursos, miden la 


\section{El Buyer Persona como factor clave entre las tendencias en Gestión Empresarial}

Vol. 3, núm. 3 Esp., (2019)

Marola Narcisa Beltrán Mora; Víctor Martín Parrales Carvajal; Gerson Damacio Ledesma Álvarez

competitividad según su participación relativa en el mercado o su rentabilidad y utilizan

la estrategia de la competitividad para mejorar su desempeño. (p. 3).

Ahora, respecto a la competitividad empresarial, los mismos citan a Mitchelmore y Rowley, debido a fueron quienes argumentaron que "(...) se considera importante para el crecimiento del negocio y el éxito, donde es factor clave el desarrollo de competencias conceptuales y de relación en la gerencia, asociados a la creación de estrategias empresariales." (p. 3).

Ya concretando el marco teórico de su investigación, fundamentados en Aguilar et al. refieren que:

La administración estratégica en su concepto visionario es un conjunto de técnicas e instrumentos utilizados preferentemente en las empresas para llevar a cabo un crecimiento, desarrollo y consolidación de las mismas utilizando diversas herramientas que sean eficaces para poder lograr el objetivo, utilizando modelos de competencia innovadores para revisar en detalle, qué fortalezas, debilidades, oportunidades o amenazas tienen para competir en los mercados nacionales e internacionales. (p. 4).

Entonces. Teniendo claro los conceptos antes referidos, es más sencillo comprender que "la gestión empresarial pone el énfasis, no tanto en la organización de los recursos, como en la planificación de los procesos para llevar a cabo los objetivos de la corporación." (Escuela Europea de Management, 2016). 


\section{El Buyer Persona como factor clave entre las tendencias en Gestión \\ Empresarial}

Vol. 3, núm. 3 Esp., (2019)

Marola Narcisa Beltrán Mora; Víctor Martín Parrales Carvajal; Gerson Damacio Ledesma

Álvarez

Gestión Tecnológica como factor de la competitividad empresarial

De Hidalgo \& Fernández (2014), se comprende que, fue a partir de la década de los años setenta cuando en varias regiones del mundo se fueron dando una serie de fenómenos macroeconómicos tales como: la ruptura del Sistema Monetario Internacional con la primera devaluación del dólar; la crisis petrolera en 1973; la acentuación de la saturación de los mercados de bienes de consumo duradero a raíz de la recesión de la demanda provocada por dicha crisis del petróleo; el elevado proceso inflacionario en los países desarrollados y el surgimiento de nuevos polos industriales y tecnológicos en diversos países del sudeste asiático, con empresas capaces de utilizar tecnologías avanzadas de producción con ventajas comparativas de costes; lo que hizo que:

La electrónica se convierte en la tecnología clave de la diversificación y el mercado de nuevos productos informáticos en el mercado de mayor crecimiento. Las "tecnologías de producto" se convierten de esta forma en un factor estratégico del desarrollo empresarial. [...] La automatización y la informatización de los procesos productivos y administrativos colocan a las nuevas tecnologías en el centro de atención de las estrategias empresariales, orientadas a la reducción de costes y a los incrementos de productividad. [...] o...bligan a las empresas de los países desarrollados a tratar de sustituir políticas de competencia basadas en el precio por políticas de competencia basadas en factores "intangibles", en las que la calidad, el diseño, el servicio postventa y, principalmente, la innovación tecnológica comienza a ser considerados como factores claves de la competitividad y, por tanto, componentes básicos de las nuevas políticas 


\section{El Buyer Persona como factor clave entre las tendencias en Gestión Empresarial}

Vol. 3, núm. 3 Esp., (2019)

Marola Narcisa Beltrán Mora; Víctor Martín Parrales Carvajal; Gerson Damacio Ledesma Álvarez

industriales. (p. 32-33).

Así mismo, el tratadista agrega que la gestión adecuada de la innovación tecnológica es significativa en la empresa, y paralelamente, la capacidad que esta tenga de innovar, se convierte en un recurso adicional de la misma, tanto como el de su capacidad productiva, financiera y comercial, de allí que entonces ello deba ser tratado de una manera eficiente y detallada.

Por gestión de la innovación se entiende el proceso orientado a organizar y dirigir los recursos disponibles, tanto humanos como técnicos y económicos, con el objetivo de aumentar la creación de nuevos conocimientos, generar ideas que permitan obtener nuevos productos, procesos y servicios o mejorar los existentes, y transferir esas mismas ideas a las fases de fabricación y comercialización. Por ello, las medidas de estímulo a la innovación no deben centrarse solamente en las primeras fases del proceso, sino que deben llegar hasta constituirse en apoyos importantes a la comercialización de los nuevos productos o tecnologías desarrolladas, tanto en mercados nacionales como internacionales. (p. 34).

\section{Marketing Estratégico}

Primeramente, se considera importante dejar claro que el marketing empresarial puntualiza:

qué estrategias y tácticas deben implementarse para lograr todos los objetivos anteriores a través de la imagen y los mensajes que la empresa transmite al exterior. [...] es el encargado de intermediar entre esta cultura: misión, visión, valores y la estrategia comercial de la empresa. [...] trata de vender productos y servicios en concreto a través 


\section{El Buyer Persona como factor clave entre las tendencias en Gestión \\ Empresarial}

Vol. 3, núm. 3 Esp., (2019)

Marola Narcisa Beltrán Mora; Víctor Martín Parrales Carvajal; Gerson Damacio Ledesma

Álvarez

de la imagen y los mensajes que transmite la compañía (estando estos alineados con su misión y visión). (Gestion.org, 2018).

Esta misma fuente afirma que existen varios tipos de estrategias de marketing empresarial y entre esas están:

- Experiencia publicitaria: significa cómo de positiva o negativa es la experiencia de la persona que recibe una acción de marketing. Una publicidad intrusiva se asociará de inmediato con valores negativos, mientras que una publicidad que ni lo parece tendrá mucho mejores resultados. Outbound marketing vs Inbound marketing.

- Imagen de producto: aunque el refranero diga que no debemos juzgar un libro por su portada, la realidad es que sí lo hacemos. Una imagen cuidada transmite profesionalidad y calidad. Echa un vistazo a nuestra sección de branding.

- Atención al cliente: uno de los factores más importantes y, a menudo, más descuidados. Un mal servicio penaliza gravemente por muy bueno que sea el producto.

- Patrocinios: ¿qué valores se asocian a los eventos que patrocina tu empresa? En nuestro artículo sobre marketing deportivo hablamos del caso del torneo de Wimbledon, y cómo los sponsors buscan anunciarse por los valores que transmite.

- Pricing: la estrategia de precios de una marca, implícitamente, puede decir qué tipo de empresa es. Te imaginarás que una compañía lowcost no utiliza la misma estrategia que una de artículos de lujo. 


\section{El Buyer Persona como factor clave entre las tendencias en Gestión Empresarial}

Vol. 3, núm. 3 Esp., (2019)

Marola Narcisa Beltrán Mora; Víctor Martín Parrales Carvajal; Gerson Damacio Ledesma Álvarez

- Punto de venta: volviendo al ejemplo de Apple, si visitas una de sus tiendas enseguida notarás qué pretende transmitir la marca: locales espaciosos, elegantes diseños minimalistas... Si quieres saber más lee nuestro artículo sobre trade marketing o merchandising.

- Imagen online: no sólo la página web, las redes sociales se han convertido en las grandes aliadas del marketing empresarial. A golpe de clic permiten transmitir de forma visual qué es y qué quiere conseguir una organización. ¿Eres un freelance o autónomo? Echa un ojo a nuestra guía de marca personal.

- Marketing social: las acciones altruistas son generadoras de confianza inmediata. Atento a nuestro artículo sobre marketing social y desarrollo sustentable. (Gestion.org, 2018).

Corrales (2011) en su afirmó:

La planificación estratégica es la que mantiene a la empresa en constante adaptación a su entorno. Lo hace anticipándose a los cambios y contrarrestando los efectos negativos del mismo con la máxima utilización de sus fortalezas, aprovechando también las oportunidades y reduciendo las debilidades a fin de protegerse de las amenazas. Es una manera de mirar hacia el futuro, proyectándose de una manera preconcebida. La mayoría de los autores coinciden en que la función del marketing estratégico es orientar a la empresa hacia las oportunidades económicas que ofrecen un potencial de crecimiento y rentabilidad. 


\section{El Buyer Persona como factor clave entre las tendencias en Gestión \\ Empresarial}

Vol. 3, núm. 3 Esp., (2019)

Marola Narcisa Beltrán Mora; Víctor Martín Parrales Carvajal; Gerson Damacio Ledesma

Álvarez

Seguidamente, el mismo refiere que:

Rafael Muñiz plantea también que una de las características más útiles e importantes del marketing consiste en poder planificar, con bastante garantía de éxito, el futuro de una empresa, basándose para ello en las respuestas que se ofrecen a las demandas del mercado. Considerando que el entorno en el que hay que posicionarse cambia y evoluciona constantemente, el éxito de la empresa dependerá, en gran parte, de la capacidad de adaptación y anticipación a estos cambios. Hay que ser capaz de comprender en qué medida y de qué forma los cambios futuros que experimentará el mercado afectarán a la empresa y de establecer las estrategias más adecuadas para aprovecharlos al máximo en beneficio propio. Por tanto, concluye Muñiz que "el marketing estratégico busca conocer las necesidades actuales y futuras de los clientes, localizar nuevos nichos de mercado, identificar segmentos de mercado potenciales, valorar el potencial e interés de esos mercados, orientar a la empresa en busca de esas oportunidades y diseñar un plan de actuación que consiga los objetivos buscados. En este sentido y motivado porque las compañías actuales se mueven en un mercado altamente competitivo se requiere, del análisis continuo de las diferentes variables del DAFO, no sólo de la empresa sino también de la competencia en el mercado. En este contexto las empresas en función de sus recursos y capacidades deberán formular las correspondientes estrategias de marketing que les permitan adaptarse a dicho entorno y adquirir ventaja a la competencia. Así pues, el marketing estratégico es indispensable para que la empresa pueda, no sólo sobrevivir, sino posicionarse en un lugar destacado 


\section{El Buyer Persona como factor clave entre las tendencias en Gestión Empresarial}

Vol. 3, núm. 3 Esp., (2019)

Marola Narcisa Beltrán Mora; Víctor Martín Parrales Carvajal; Gerson Damacio Ledesma Álvarez

en el futuro. (Corrales, 2011).

Buyer Persona

Siquieira, siendo citado en la obra de García, Pabón, Patiño, Plata \& Soltau (2019) es un personaje ficticio que se construye a partir de la etnografía de una población (edad, sexo, costumbres, creencias, entre muchas otras), con perfil psicológico, calidades y comportamientos similares. (p. 14-15).

Valdés (2019) como experto en el área ofrece una amplia y detallada exposición de lo implica el buyer persona. Antes que todo, da a entender que se trata de la construcción un modelo de cliente idóneo de un producto o servicio que, teniendo en cuenta datos sociodemográficos precisos, así como también información sobre aspectos tales como conducta online, personal, profesional y de la relación con la empresa que ofrece el producto o servicio, puede llegar a ser uno de los pilares transcendentales en el inbound marketing (entendido como la herramienta para producir contenido de valor que despierte el interés de la audiencia objetivo) y no solamente por el hecho de conocer las inquietudes, intereses y dolores que pueda tener ese cliente al que se quiere llegar, sino además, se traduce en el cómo y dónde ese cliente busca el producto o servicio.

Desde la perspectiva de un proveedor de producto o servicio, sigue explicando destacadamente que:

Una de las características más importantes para el buyer persona (que además suele ser el principal diferenciador entre un buyer persona y otro) es el dolor o también 


\section{El Buyer Persona como factor clave entre las tendencias en Gestión \\ Empresarial}

Vol. 3, núm. 3 Esp., (2019)

Marola Narcisa Beltrán Mora; Víctor Martín Parrales Carvajal; Gerson Damacio Ledesma

Álvarez

llamado pain. Aunque el término "dolor" pueda parecer inadecuado, en realidad llamamos así a cualquier necesidad, motivación o preocupación que tenga el buyer persona y que pueda ser solucionada con nuestro producto o servicio. Si conocemos el dolor o pain de nuestro cliente ideal, conocemos algo muy valioso; su driver, la motivación que le impulsa a hacer una determinada búsqueda en Google o le predispone a comprar lo que le ofrece nuestra empresa. Por ello, es muy importante tener una imagen mental muy clara de nuestro buyer persona. Para lograrla, necesitamos reunir mucha más información, que no sólo nos ayudará a crear el contenido con el enfoque adecuado, sino la estrategia de contenido de forma global, la imagen de marca y la selección de canales a través de la que haremos la difusión. ¿De qué información se trata?

- Personal: aficiones, personas que influencian en sus decisiones, qué le hace feliz, cuáles son sus objetivos, qué motivaciones tiene, etc.

- Conducta online: qué redes sociales utiliza, si compra productos online y de qué tipo, dónde busca la información, con qué frecuencia lo hace, cuáles son sus páginas de referencias, en qué horas está online, etc.

- Conducta laboral: responsabilidades, retos, mayores influencias, etc.

- Relación con nuestra compañía: de qué nos conoce, motivos por los que nos ha contratado o comprado, cómo nos conoció, qué es lo que valora más de nosotros como empresa, qué interacción tiene con la marca, etc. 


\section{El Buyer Persona como factor clave entre las tendencias en Gestión Empresarial}

Vol. 3, núm. 3 Esp., (2019)

Marola Narcisa Beltrán Mora; Víctor Martín Parrales Carvajal; Gerson Damacio Ledesma Álvarez

Continúa Valdés ampliando su exposición al detallar que existen tres perfiles de buyer persona que son:

- Decisor: es la persona que puede tomar la decisión final de la compra. Acostumbra a ser el caso más extendido, especialmente en B2C.

- Prescriptor: la persona que recomienda el producto. Un ejemplo muy claro podría ser el de un médico que puede ser el prescriptor de un determinado medicamento.

- Influenciador: la persona que, con su opinión, puede condicionar positiva o negativamente la decisión de compra. Es muy importante saber quiénes son los influencers de nuestro buyer persona, ya que esto nos ayudará a decidir con qué blogs y perfiles en las redes sociales vale la pena contactar para conseguir algún tipo de colaboración.

\section{Creación de un Buyer Persona}

Una vez identificados y clasificados los buyer persona según los criterios, necesidades y expectativas de la empresa, es preciso tener en cuenta los pasos para crearlos:

- Identificar qué información necesitamos para desarrollar los buyer personas.

- Determinar cómo vamos a investigar los buyer personas y cómo obtendremos las respuestas a las preguntas que hemos formulado.

- Realizar esta investigación, recopilando información y respuestas, y anotar en la 


\section{El Buyer Persona como factor clave entre las tendencias en Gestión \\ Empresarial}

Vol. 3, núm. 3 Esp., (2019)

Marola Narcisa Beltrán Mora; Víctor Martín Parrales Carvajal; Gerson Damacio Ledesma

Álvarez

hoja de desarrollo del buyer persona las respuestas mayoritarias.

- Usar una hoja de desarrollo del buyer persona para cada perfil que tengamos.

- Convertir la hoja de desarrollo en un perfil completo de buyer persona, usando las buenas prácticas de desarrollo de buyer personas.

- Contar la historia completa del buyer persona siguiendo las buenas prácticas y el perfil completo. (Valdés, 2019).

Identificar qué información necesitamos para desarrollar los buyer personas

López (2015) propone una plantilla para definir un buyer persona:

1. Nombre: aunque el Buyer Persona es una representación semi ficticia del cliente ideal de una empresa, es conveniente ponerle nombre para que el equipo interiorice sus rasgos, sus objetivos y necesidades y lo recuerde con mayor facilidad. Normalmente se le añade un apellido relacionado una actividad que lo defina.

2. Antecedentes o background: reúne los rasgos personales y profesionales que definan la vida del Buyer Persona como por ejemplo su trabajo, familia, estudios, etc.

3. Información demográfica: aquí se exponen los datos relativos al sexo, la edad, localización y otros temas relacionado con la segmentación del mercado. 


\section{El Buyer Persona como factor clave entre las tendencias en Gestión Empresarial}

Vol. 3, núm. 3 Esp., (2019)

Marola Narcisa Beltrán Mora; Víctor Martín Parrales Carvajal; Gerson Damacio Ledesma Álvarez

4. Identificadores: en este punto se definen las fuentes de comunicación más utilizadas por el Buyer Persona y su actitud a la hora de mantener una relación con la empresa.

5. Objetivos: recoge los objetivos primarios y secundarios del Buyer Persona.

6. Desafíos: se definen los retos a los que el Buyer Persona debe hacer frente para alcanzar los objetivos pretendidos.

7. ¿Qué podemos hacer?: en este punto se proporcionan ideas con las que la empresa puede ayudar a que el Buyer Persona logre sus objetivos.

8. Citas reales: se pueden recoger también frases reales de clientes o clientes potenciales que ayuden a entender mejor sus necesidades y sentimientos.

9. Objetivos comunes: aquí se concreta por qué un usuario debería adquirir el producto o servicio de la empresa y no en su competencia.

10. Mensajes de marketing: en este punto se hace hincapié en cómo debe describir la empresa su solución a su Buyer Persona, es decir, cómo enfocar y qué palabras utilizar para que el Buyer Persona se fije en las ofertas de la empresa.

11. Elevator pitch: despertar el interés del Buyer Persona sobre el producto o servicio de la compañía en unas pocas frases. (p. 12, 13). 


\section{El Buyer Persona como factor clave entre las tendencias en Gestión \\ Empresarial}

Vol. 3, núm. 3 Esp., (2019)

Marola Narcisa Beltrán Mora; Víctor Martín Parrales Carvajal; Gerson Damacio Ledesma

Álvarez

Métodos para Investigar los buyer personas

- Entrevistas a clientes (presencialmente, por videoconferencia o por teléfono o a través de algún departamento que tenga contacto con el cliente)

- Herramientas de inteligencia de leads

- Entrevistas online

- Llamadas telefónicas

- Focus Group

- Encuesta offline

\section{Tendencia de Buyer Persona como estrategia de gestión empresarial}

Tratar de comprender como se ha llegado hoy en día en la gestión empresarial a considerar estrategias basadas en el cliente, cabría mencionar entonces aportes como el de Valenzuela y Torres (2008) quienes con su obra han aportado una perspectiva respecto a los antecedentes de la orientación al valor del cliente, puesto que indican que este surge:

Como respuesta al entorno cada vez más turbulento, competitivo y exigente, tanto del mercado como en tecnología, la orientación al mercado y el marketing relacional convergen en la estrategia de negocio basada en la gestión de relaciones con clientes (Customer Relationship Management, CRM) en función del valor del tiempo de vida de la relación cliente-empresa. (p. 68). 


\section{El Buyer Persona como factor clave entre las tendencias en Gestión Empresarial}

Vol. 3, núm. 3 Esp., (2019)

Marola Narcisa Beltrán Mora; Víctor Martín Parrales Carvajal; Gerson Damacio Ledesma Álvarez

De los mismos, se hace posible comprender que importantes estudios desarrollados entorno a estrategias como estas, han arrojado valiosos resultados que advierten que "que las compañías deberían valorar la cartera de clientes como el activo clave para conseguir la rentabilidad del negocio" (p. 68). Con base en la concepción de Reinartz, Krafft y Hoyer sobre CRM, dicen que:

Este paradigma sitúa al cliente en el centro de todo el negocio, en este sentido, la gestión integrada de la relación con el cliente se yergue como estrategia básica de supervivencia y crecimiento. [...] Al centrarse en el cliente, éste pasa a estar por delante de los procesos internos de la organización, siendo la forma en que el cliente quiere interactuar con ella lo prioritario. De esta manera el actuar para el cliente pasa a ser substituido por actuar con el cliente. (p. 68-69).

Evidentemente, se tiene claro que el buyer persona no es una herramienta tan versátil que por sí sola sea suficiente como para marcar una tendencia en el área de la gestión empresarial, porque, de hecho, se reitera que esta se le considera previa y hasta trascendentalmente para la ejecución de las estrategias de Inbound marketing, Sin embargo, han sido varios equipos de expertos en el tema los que inclusive desde 2018 venían percatándose del uso de este tipo de herramientas, tales como Data Crush (2019) quienes afirmaron:

...hemos observado cambios importantes en el camino de compra y en las expectativas de los consumidores a lo largo de este camino. Quieren comodidad y ultrapersonalización. En el 2019 debemos ser expertos en “audiencias". Pero antes de definir una audiencia, debemos tener claro cuál es nuestro objetivo. Como las preferencias de 


\section{El Buyer Persona como factor clave entre las tendencias en Gestión \\ Empresarial}

Vol. 3, núm. 3 Esp., (2019)

Marola Narcisa Beltrán Mora; Víctor Martín Parrales Carvajal; Gerson Damacio Ledesma

Álvarez

los consumidores y los comportamientos de compra cambian constantemente, la definición del "cliente tipo" o "Buyer Persona" debe ser flexible. La estrategia de marketing de las marcas depende fuertemente de la definición del o los Buyer Persona.

Puede parecer una recomendación básica, pero de la misma manera que debemos analizar y evaluar nuestra estrategia regularmente, nuestro Buyer Persona debería seguir el mismo ritmo... si no es más seguido aún. (Data Crush, 2019).

Otro equipo que antes de finalizar el primer trimestre del corriente igualmente se percató de esta directriz han sido los de IE Avanzado, puesto que al respecto recordaron que ya desde 2013 el icónico Jobs [de Apple] advertía la necesidad de partir de la observación de "la experiencia del cliente para diseñar la tecnología" siendo entonces precisamente en 2019 que tal idea adquiere cierta notoriedad al perfilarse:

...como una de las mayores tendencias de gestión empresarial. Por tanto, los emprendedores deben asegurarse de que el proceso de desarrollo de productos comience con la investigación de la buyer persona y se centre en brindar una experiencia personalizada que realmente aporta valor a los clientes". (IE Avanzado, 2019).

\section{Conclusiones.}

Del análisis de los resultados y por consiguiente de la argumentación experimentada varios entendidos en la materia, es consecuente comprender y deducir que existe basta evidencia que sustenta la teoría de que, definir un Buyer Persona resulta ser un elemento clave previo al establecimiento de estrategias de Inbound Marketing, ya que es indiscutible la obtención de 


\section{El Buyer Persona como factor clave entre las tendencias en Gestión Empresarial}

Vol. 3, núm. 3 Esp., (2019)

Marola Narcisa Beltrán Mora; Víctor Martín Parrales Carvajal; Gerson Damacio Ledesma Álvarez

mejores resultados.

De hecho, se comparte la creencia de que la ideología del inbound representará una de las maniobras más sostenibles a largo plazo en un futuro próximo, ya que, independientemente de la que acelerada evolución del consumidor, esa valiosa información significará posiblemente en una de las muy pocas formas de construir relaciones comerciales respetables y de estar al tanto de las empresas.

\section{Bibliografía.}

Alguacil, M., Crespo, J., \& Pérez, C. (2019). Análisis sociodemográfico de la percepción de marca en un servicio deportivo público: del target al buyer persona. Retos: Nuevas tendencias en educación física, deporte y recreación, 37, 139-146.

Cafaro Malavé, R. (2019). El comprador ideal, una estrategia de marketing para las PYMES modernas. Business Innova Sciences, 1(1), 70-79.

Corrales, Y. (2011). El Marketing Estratégico como base para potenciar la exportación en las empresas cubanas. Observatorio de la Economía Latinoamericana(152), 1-25 .

Data Crush. (28 de Marzo de 2019). Las 10 tendencias que van a marcar em marketing en el 2019. Recuperado el 05 de 11 de 2019, de Data Crush: https://www.datacrush.la/blog/10tendencias-que-van-a-marcar-el-marketing-2019/

Escuela Europea de Management. (30 de Septiembre de 2016). Diferencias entre dirección, gestión y administración de empresas. Obtenido de Escuela Europea de Management: http://www.escuelamanagement.eu/direccion-de-empresas/diferencias-entre-direcciongestion-y-administracion-de-empresas

Espejel, J., Leyva, A., \& Cavazos, J. (2017). La Competitividad Emprsarial medida a través de la Admiinistración Estratégica y Planeación Estratégica. Un análisis comparativo para la pequeña y mediana empresa. XXII Congreso Internacional de Contaduría, Administración e Informática (pág. 26 ). Ciudad de México: Universidad Naional Autónoma de México. Recuperado el 05 de 11 de 2019, de http://congreso.investiga.fca.unam.mx/docs/xxii/docs/1.04.pdf 


\section{EI Buyer Persona como factor clave entre las tendencias en Gestión \\ Empresarial}

Vol. 3, núm. 3 Esp., (2019)

Marola Narcisa Beltrán Mora; Víctor Martín Parrales Carvajal; Gerson Damacio Ledesma

Álvarez

García, L., Pabón, M., Patiño, D., Plata, K., \& Soltau, S. (2019). Estrategias digitales para Negocios. Universidad del Rosario. Bogotá: Escuela de Administración. Recuperado el 05 de $11 \quad$ de $2019, \quad$ de https://repository.urosario.edu.co/bitstream/handle/10336/20402/Garc\%c3\%adaD\%c3\%a daz-LauraSof\%c3\%ada.pdf?sequence $=4 \&$ isAllowed $=\mathrm{y}$

Gestion.org. (2018). Marketing empresarial: concepto, ejemplos y clases. Obtenido de Gestion: https://www.gestion.org/internacionalizacion-de-una-empresa/

Hidalgo, A., \& Fernández, M. (2014). Gestión de la Innovación Tecnológica como respeuesta estartégica a los paradigmas. Vectores de Investigación, 9(9), 31-51.

IE Avanzado. (18 de Marzo de 2019). Siete tendencias de gestión empresarial en 2019. Obtenido de IE Avanzado: https://ieavanzado.com/blog/gestion-de-empresas/tendencias-gestionempresarial-en-2019

López Martínez, I. (2015). Diseño metodológico de definición de público objetivo para benchmarking web en el sector de la moda española. Universidad Politécnica de Valencia, Escuela Técnica Superior de Ingeniería en Informática. Valencia: Escuela Técnica Superior de Ingeniería en Informática.

Valdés, P. (25 de Abril de 2019). Buyer persona: el factor clave en tu estrategia de marketing y ventas. Recuperado el 27 de Noviembre de 2019, de InboundCycle: https://www.inboundcycle.com/buyer-persona

Valenzuela, L., \& Torres, E. (2008). gestion empresarial orientada al valor del cliente como fuente de ventaja competitiva. Propuesta de un modelo explicativo. Estudios Gerenciales, 24(109), 65-86.

Vargas, C. A., Solarte, C., \& Moreno, I. C. (2012). La gestión empresarial ¿por qué es importante la gestión empresarial en las organizaciones modernas? Ensayos: Revista de Estudiantes de Administración de Empresas, 5(5), 246-260.

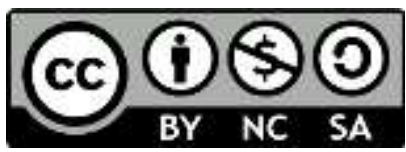

RECONOCIMIENTO-NOCOMERCIAL-COMPARTIRIGUAL

CC BY-NC-SA

ESTA LICENCIA PERMITE A OTROS ENTREMEZCLAR, AJUSTAR Y CONSTRUIR A PARTIR DE SU OBRA CON FINES NO

COMERCIALES, SIEMPRE Y CUANDO LE RECONOZCAN LA AUTORÍA Y SUS NUEVAS CREACIONES ESTÉN BAJO UNA LICENCIA

CON LOS MISMOS TÉRMINOS. 